\title{
Synthesis of novel 3-arylcyclopenta[c]quinolines via acid-induced domino cyclization of 2-arylamino-2-methylthioethenyl 2-arylcyclopropyl ketones
}

\author{
S. K. S. Yadav, A. K. Yadav, G. S. M. Sundaram, H. Ila, * and H. Junjappa \\ Department of Chemistry, Indian Institute of Technology, Kanpur-208016, India \\ E-mail:hila@iitk.ac.in
}

Dedicated to Professor Lutz F. Tietze on his 65th birthday

\begin{abstract}
A novel acid-induced domino cyclization of $N, S$-anilinoacetals of type 3 derived from 2arylcyclopropyl ketones, is reported which yields a wide range of substituted- and fused- 3arylcyclopenta $[c]$ quinolines $\mathbf{6}$ with concomitant formation of a cyclopentane and a quinoline ring.
\end{abstract}

Keywords: Domino cyclization, cyclopenta[c]quinolines, $N, S$-anilinoacetals

\section{Introduction}

Substituted quinolines and their benzo- or hetero- fused analogues represent an important class of heterocyclic compounds because of their presence in numerous biologically active natural products, along with the wide spectrum of physiological activities displayed by these class of compounds. ${ }^{1,2}$ As a consequence, much attention has been focused towards developing new synthetic methods for substituted- and fused quinolines. ${ }^{1,3}$ Domino reactions are of growing interest in synthetic organic chemistry because they allow rapid construction of polycyclic structures in a single operation and confer great strategic value in molecular construction. ${ }^{4}$ Our own interest in this regard has been concerned with the elaboration of domino reactions involving acid-induced rearrangements of 2-aryl-(or heteroaryl-)cyclopropyl ketones with an $\alpha$ (bismethylthio)-methylene functionality as the cationic cyclization terminator, which yield a range of carbocyclic products such as substituted cyclopentanones, ${ }^{5 \mathrm{a}, \mathrm{b}}$ cyclopenta[ $[b]$ indanes, ${ }^{5 \mathrm{c}-\mathrm{d}}$ diquinanes, ${ }^{5 \mathrm{~d}}$ 1-arylindanes, ${ }^{5 \mathrm{e}}$ bicyclo[3.2.1] ${ }^{\text {octene }},{ }^{5 \mathrm{f}}$ and other cyclopentano-fused heterocycles. $^{5 \mathrm{~g}-\mathrm{i}}$ In continuation of these studies, we now report the domino carbocationic cyclization of arylcyclopropyl ketones of type 3 carrying an ( $N, S$-aminomethylene) functionality, yielding 3- arylcyclopenta[c]quinolines (compound 6) in a single one-pot operation. 


\section{Results and Discussion}

The cyclopropyl ketone (compound 1) was prepared in quantative yield by treatment with dimethyloxosulphonium methylide generated from the corresponding sulphonium salt to the corresponding unsaturated ketene $S, S$-acetals in the presence of phase transfer catalyst. ${ }^{5 \mathrm{a}, 5 \mathrm{i}, 6}$ The $N, S$-arylaminoacetals (compounds $\mathbf{3 a}-\mathbf{j}$ ) required for studying this transformation were prepared by displacement of one of the methylthio groups of the corresponding $\alpha$-oxoketene dithioacetals (compound 1) by a substituted aniline in the presence of $n$-BuLi in THF. ${ }^{7}$ The structures of all these newly synthesized $N, S$-acetals (compounds $\mathbf{3 a}-\mathbf{j}$ ) were confirmed with the help of spectral and analytical data. The ${ }^{1} \mathrm{H}$ NMR spectra of these $N, S$-acetals show that they exist in the intramolecular H-bonded form $\mathbf{3 A}$.

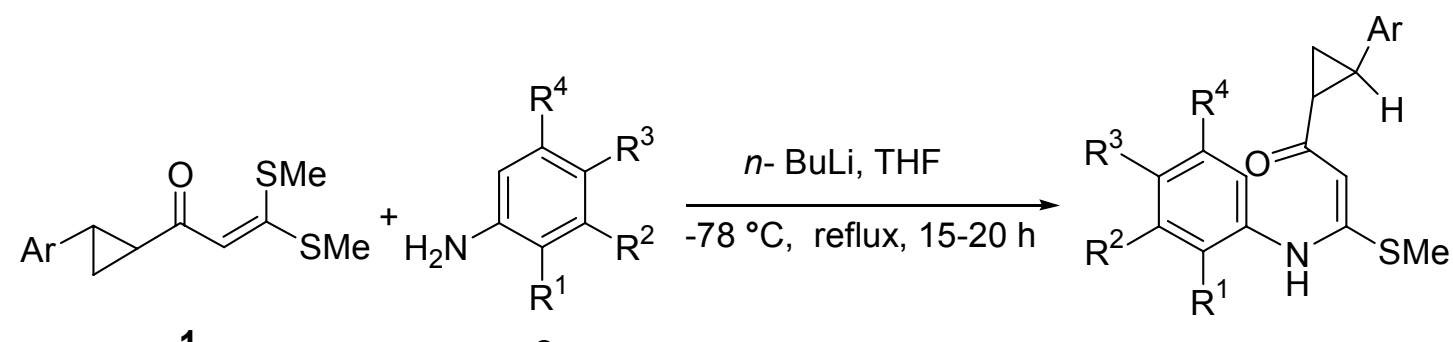

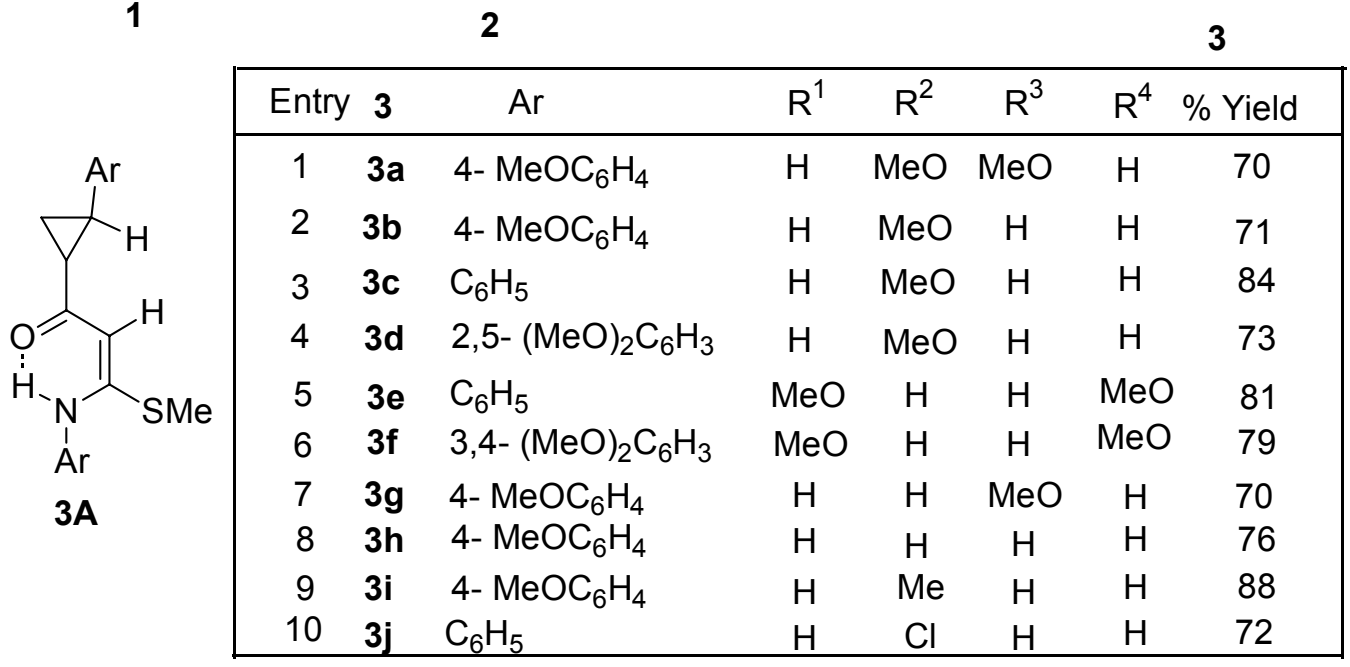

\section{Scheme 1}

The cyclization of the $N, S$-anilinoacetal (compound 3a), bearing two strongly activating methoxy groups, was first examined in the presence of various protic and Lewis acids. It was envisaged that the initial acid-assisted ring-opening of the cyclopropyl ketone (compound 3 ) would furnish the stable benzylic carbocation 4 which could be intercepted by the electron-rich $N, S$-anilino(methylene) double bond via a 5-exo process followed by intramolecular cyclocondensation of the resulting cyclopentanone $N, S$-acetal 5 to afford angularly-fused cyclopenta[ $[c]$ quinolines (compound 6) (Scheme 2, pathway a). Alternatively, the cyclopropyl 
ketone (compound 3) might undergo either stepwise or concomitant ring-opening and intramolecular cyclocondensation to give the quinoline 7 with a pendant $\alpha$-arylpropyl carbocation side chain which can be captured intramolecularly by the electron-rich 5-position of quinoline to give novel peri-fused quinoline (compound 8) (pathway b), as was observed in our previous studies. ${ }^{5 \mathrm{~g}}$ However, a detailed study of the cyclization of compound $\mathbf{3 a}$ under the influence of various Lewis and protic acids $\left(\mathrm{H}_{3} \mathrm{PO}_{4}, \mathrm{TFA}, \mathrm{PPA}, \mathrm{CF}_{3} \mathrm{SO}_{3} \mathrm{H}, \mathrm{SnCl}_{4}, \mathrm{BF}_{3} \cdot \mathrm{OEt}_{2}\right.$, $\left.\mathrm{Cu}(\mathrm{OTf})_{2}, \mathrm{TiCl}_{4}\right)$ revealed the formation of either the cyclopenta[c]quinoline (compound $\mathbf{6 a}$ ) or the cyclopentanone $N, S$-acetal 5a as exclusive products in varying yields (Table 1), thus showing that cyclization via pathway ' $a$ ' is preferred over ' $b$ '.

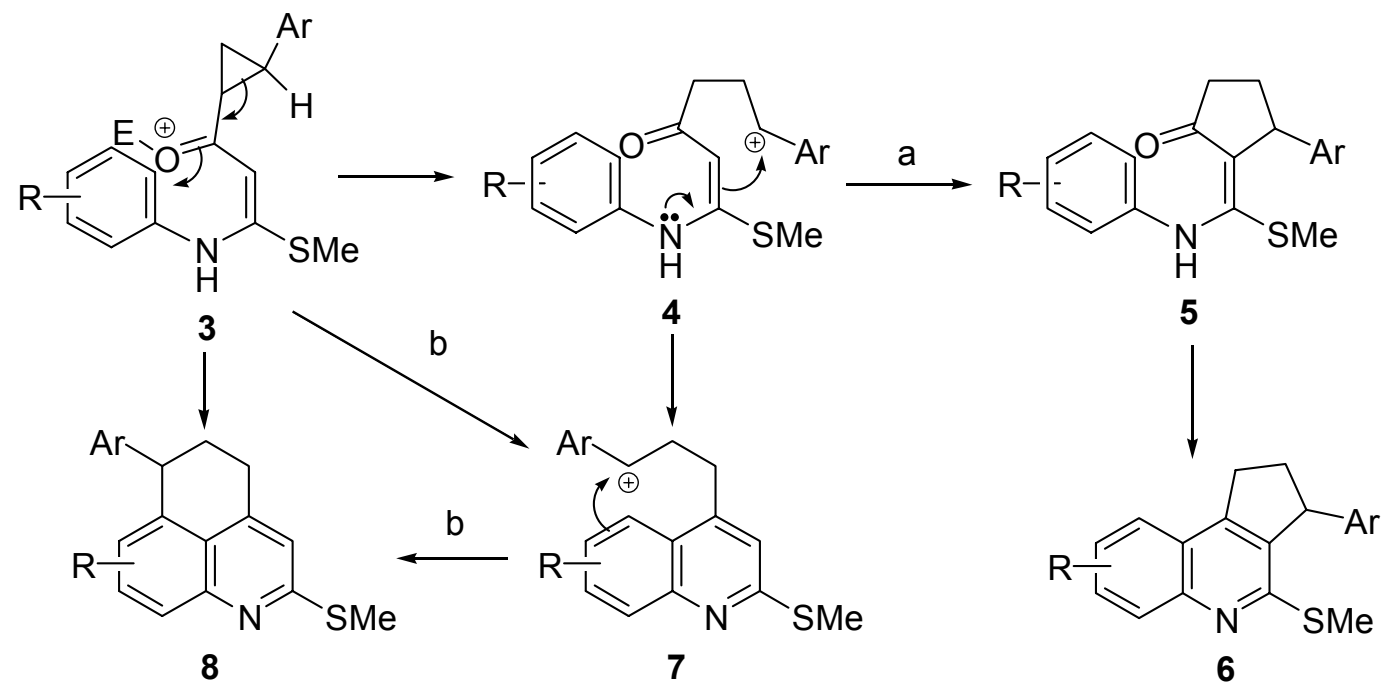

\section{Scheme 2}<smiles>COc1ccc(N/C(=C/C(=O)C2CC2Br)C(C)(C)C)cc1OC</smiles>

3a $\mathrm{Ar}=4-\mathrm{MeOC}_{6} \mathrm{H}_{4}$

$6 a$<smiles>CCC1CCC(=O)C1=C(C)Nc1ccc(OC)c(OC)c1</smiles>

$5 a$

The cyclization of compound 3a was found to be most efficient in the presence of polyphosphoric acid at $90{ }^{\circ} \mathrm{C}$; therefore all the subsequent cyclizations were carried out in the presence of this acid. Thus, Scheme 3 shows cyclization-rearrangement of the various substituted $N, S$-anilinoacetals (compounds $\mathbf{3 a}-\mathbf{j}$ ) in the presence of PPA yielding the respective substituted 3-arylcyclopenta[c]quinolines (compounds $\mathbf{6 a - j}$ ) in 46-56\% overall yields, which we were not able to improve. 
Table 1. Lewis/protic acid-induced rearrangement of the cyclopropyl ketone (3a)

\begin{tabular}{cclccc}
\hline Entry & $\mathbf{3}$ & Reaction Conditions & Time (h) & \multicolumn{2}{c}{ Products } \\
& & & & $\mathbf{6 a}$ (\% Yield) $\mathbf{5 a}$ (\% Yield) \\
\hline 1 & $\mathbf{3 a}$ & $\mathrm{H}_{3} \mathrm{PO}_{4} / 60^{\circ} \mathrm{C} / \Delta$ & 4 & 34 & - \\
2 & $\mathbf{3 a}$ & $\mathrm{PPA} / 90{ }^{\circ} \mathrm{C} / \Delta$ & 8 & 53 & - \\
3 & $\mathbf{3 a}$ & $\left(\mathrm{CF}_{3} \mathrm{SO}_{3}\right)_{2} \mathrm{Cu} / \mathrm{CH}_{2} \mathrm{Cl}_{2} / \mathrm{RT}$ & 4 & 30 & - \\
4 & $\mathbf{3 a}$ & $\mathrm{TFA} / \mathrm{CH}_{2} \mathrm{Cl}_{2} / \Delta$ & 20 & 24 & 18 \\
5 & $\mathbf{3 a}$ & $\mathrm{CF}_{3} \mathrm{SO}_{3} \mathrm{H}, \mathrm{RT}$ & 10 & 36 & 16 \\
6 & $\mathbf{3 a}$ & $\mathrm{SnCl}_{4} / \mathrm{CH}_{2} \mathrm{Cl}_{2} / 0{ }^{\circ} \mathrm{C}-\mathrm{RT}$ & 10 & - & 20 \\
7 & $\mathbf{3 a}$ & $\mathrm{SnCl}_{4} / \mathrm{C}_{6} \mathrm{H}_{6} / \mathrm{reflux}$ & 6 & 18 & 26 \\
8 & $\mathbf{3 a}$ & $\mathrm{BF}_{3} \cdot \mathrm{OEt}_{2} / \mathrm{CH}_{2} \mathrm{Cl}_{2} / 0{ }^{\circ} \mathrm{C}-\mathrm{RT}$ & 15 & - & 30 \\
9 & $\mathbf{3 a}$ & $\mathrm{BF}_{3} \cdot \mathrm{OEt}_{2} / \mathrm{C}_{6} \mathrm{H}_{6} / \mathrm{reflux}$ & 8 & 35 & - \\
10 & $\mathbf{3 a}$ & $\mathrm{TiCl}_{4} / \mathrm{CH}_{2} \mathrm{Cl}_{2} / 0{ }^{\circ} \mathrm{C}-\mathrm{RT}$ & 10 & - & 30 \\
\hline
\end{tabular}<smiles>[R]c1c([R])c([R])c(N/C(C)=C/C(=O)C2CC2([3H])[AlH2])c([R])c1[R]</smiles>

$3 a-j$<smiles>[R]c1nc2c([R])c([R3])c3c(c2c([R])c1[R])CCC3[Al]</smiles>

6a-j

\begin{tabular}{|cclccccc|}
\hline Entry & $\mathbf{6}$ & \multicolumn{1}{c}{$\mathrm{Ar}$} & $\mathrm{R}^{1}$ & $\mathrm{R}^{2}$ & $\mathrm{R}^{3}$ & $\mathrm{R}^{4}$ & $\%$ Yield \\
\hline 1 & $\mathbf{6 a}$ & $4-\mathrm{MeOC}_{6} \mathrm{H}_{4}$ & $\mathrm{H}$ & $\mathrm{MeO}$ & $\mathrm{MeO}$ & $\mathrm{H}$ & 54 \\
2 & $\mathbf{6 b}$ & $4-\mathrm{MeOC}_{6} \mathrm{H}_{4}$ & $\mathrm{H}$ & $\mathrm{MeO}$ & $\mathrm{H}$ & $\mathrm{H}$ & 56 \\
3 & $\mathbf{6 c}$ & $\mathrm{C}_{6} \mathrm{H}_{5}$ & $\mathrm{H}$ & $\mathrm{MeO}$ & $\mathrm{H}$ & $\mathrm{H}$ & 55 \\
4 & $\mathbf{6 d}$ & $2,5-(\mathrm{MeO})_{2} \mathrm{C}_{6} \mathrm{H}_{3}$ & $\mathrm{H}$ & $\mathrm{MeO}$ & $\mathrm{H}$ & $\mathrm{H}$ & 54 \\
5 & $\mathbf{6 e}$ & $\mathrm{C}_{6} \mathrm{H}_{5}$ & $\mathrm{MeO}$ & $\mathrm{H}$ & $\mathrm{H}$ & $\mathrm{MeO}$ & 51 \\
6 & $\mathbf{6 f}$ & $3,4-\left(\mathrm{MeO}_{2}\right)_{6} \mathrm{C}_{6} \mathrm{H}_{3}$ & $\mathrm{MeO}$ & $\mathrm{H}$ & $\mathrm{H}$ & $\mathrm{MeO}$ & 54 \\
7 & $\mathbf{6 g}$ & $4-\mathrm{MeOC}_{6} \mathrm{H}_{4}$ & $\mathrm{H}$ & $\mathrm{H}$ & $\mathrm{MeO}$ & $\mathrm{H}$ & 50 \\
8 & $\mathbf{6 h}$ & $4-\mathrm{MeOC}_{6} \mathrm{H}_{4}$ & $\mathrm{H}$ & $\mathrm{H}$ & $\mathrm{H}$ & $\mathrm{H}$ & 52 \\
9 & $\mathbf{6 i}$ & $4-\mathrm{MeOC}_{6} \mathrm{H}_{4}$ & $\mathrm{H}$ & $\mathrm{Me}$ & $\mathrm{H}$ & $\mathrm{H}$ & 46 \\
10 & $\mathbf{6 j}$ & $\mathrm{C}_{6} \mathrm{H}_{5}$ & $\mathrm{H}$ & $\mathrm{Cl}$ & $\mathrm{H}$ & $\mathrm{H}$ & 52 \\
\hline
\end{tabular}

Scheme 3 
The corresponding benzo[ $h]$ cyclopenta[ $[c]$ quinoline (compound 6k) could also be synthesized in moderate yield (48\%) by acid induced domino- cyclization of the corresponding cyclopropyl $N, S$-acetal (compound 3k) derived from $\alpha$-naphthylamine under identical conditions (Scheme 4).

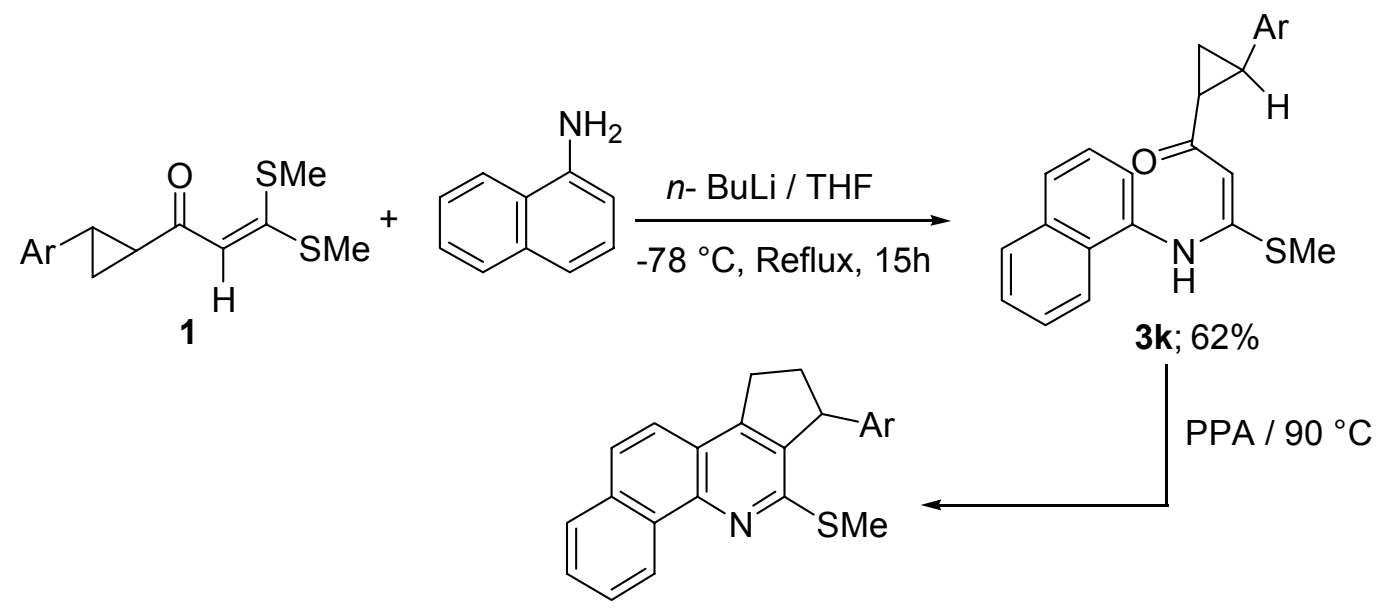

6k, $\mathrm{Ar}=4-\mathrm{MeOC}_{6} \mathrm{H}_{6}, 48 \%$

\section{Scheme 4}

Two of the 4-methylthiocyclopenta[c]quinolines (compounds $\mathbf{6 a}$ and $\mathbf{6 j}$ ) were dethiomethylated in the presence of Raney nickel to afford sulfur-free cyclopenta[c]quinolines $7 \mathbf{a}$ and $7 \mathbf{j}$ in high overall yields (Scheme 5).

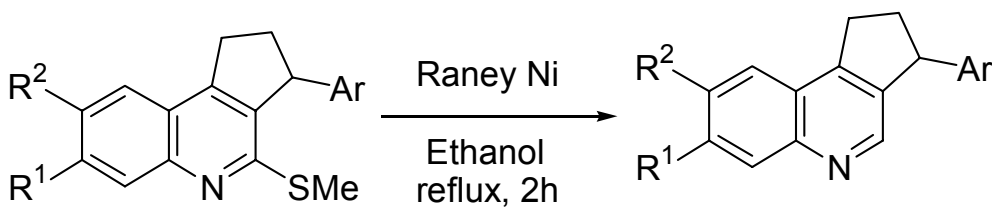

6a, 6j

7a, $\mathrm{R}^{1}=\mathrm{R}^{2}=\mathrm{MeO} ; \mathrm{Ar}=4-\mathrm{MeOC}_{6} \mathrm{H}_{4} ; 88 \%$

$7 \mathbf{j}, \mathrm{R}^{1}=\mathrm{Cl} ; \mathrm{R}^{2}=\mathrm{H} ; \mathrm{Ar}=\mathrm{C}_{6} \mathrm{H}_{5} ; 64 \%$

\section{Scheme 5}

Finally, we have also attempted ruthenium tetraoxide- mediated oxidative degradation of the 3- arylcyclopenta[c]quinoline (compound $\mathbf{6 j}$ ) with a view to obtaining the corresponding cyclopenta[c]quinoline 3-carboxylic acid (compound 8j) (Scheme 6). However, analysis of the reaction mixture showed the formation only of sulfone (compound $\mathbf{9 j}$ ) instead of compound $\mathbf{8 j}$, which could not be obtained even when excess of catalyst ( 8 mol. \%) was taken. Our attempts in this direction are still in progress. 


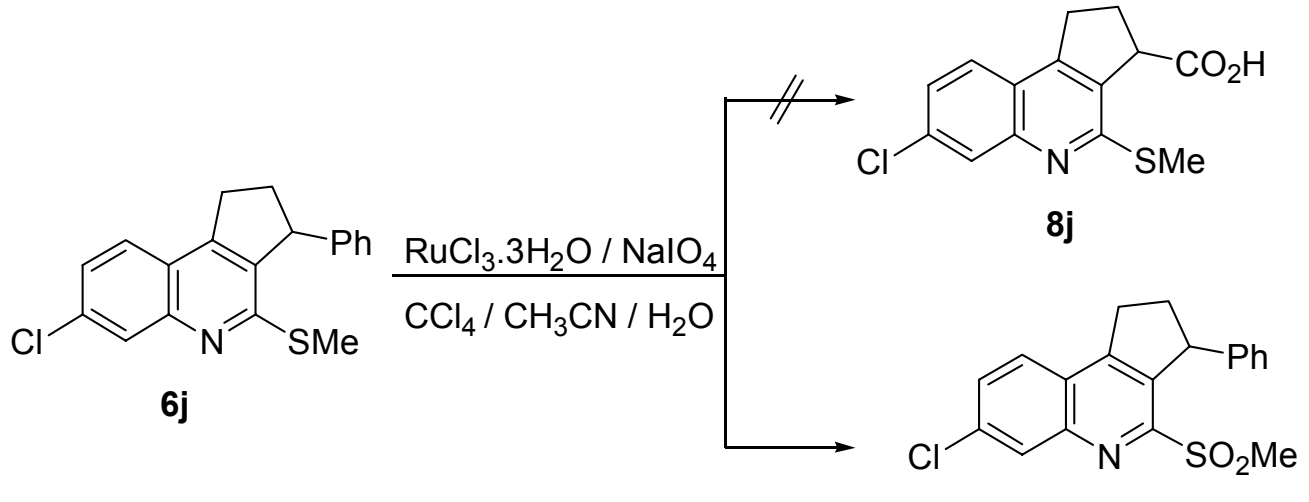

9j; $68 \%$

\section{Scheme 6}

\section{Conclusions}

In summary, we have shown that N,S-anilinoacetals (compound 3) derived from 2arylcyclopropyl ketones undergo acid-induced intramolecular domino cyclization, yielding novel substituted 3-aryl cyclopenta[c]quinolines (compound 6) in 46-56\% overall yield with concomitant formation of a cyclopentane and quinoline ring in a single operation.

\section{Experimental Section}

General Procedures. ${ }^{1} \mathrm{H}$ NMR (400 MHz) and ${ }^{13} \mathrm{C}$ NMR (100 MHz) spectra (JEOL JNM LA400) were recorded in $\mathrm{CDCl}_{3}$. TMS was used as internal reference. Melting points were obtained on Mel-Temp melting point apparatus (capillary method) and were uncorrected. The IR spectra were recorded on a Perkin Elmer 1320 spectrophotometer. The FAB mass spectra were recorded on a JEOL SX 102/DA-6000 Mass Spectrometer/Data System. Elemental analyses of the compounds were obtained using an Elementar Vario EL III Carla Erba 1108 model. Column chromatography used 100-200 mesh silica gel obtained from Acme Synthetic Chemicals (India). Substituted anilines, $\mathrm{BF}_{3} \cdot \mathrm{OEt}_{2}, \mathrm{SnCl}_{4}, \mathrm{TFA}, \mathrm{H}_{3} \mathrm{PO}_{4}(88 \%), \mathrm{Cu}(\mathrm{OTf})_{2}$ and triflic acid were purchased from commercial sources. The cyclopropyl ketone 1 was prepared according to the reported procedure. ${ }^{5 \mathrm{a}}$

\section{General procedure for preparation of $\boldsymbol{N}, S$-arylaminoacetals $(3 a-k)$}

To a stirred solution of aniline $(10 \mathrm{mmol})$ in dry THF $(20 \mathrm{~mL}), n$-BuLi $(15 \mathrm{mmol})$ was added, dropwise, under argon, over a period of $20 \mathrm{~min}$. at $-78{ }^{\circ} \mathrm{C}$ and the reaction mixture was stirred for $30 \mathrm{~min}$. at room temperature. It was cooled to $0{ }^{\circ} \mathrm{C}$, followed by addition of ketene $S$, $S$-acetal $(10 \mathrm{mmol})$ in dry THF $(20 \mathrm{~mL})$. The reaction mixture was further stirred at room temperature for 
45 minutes followed by refluxing $\left(12-15 \mathrm{~h}\right.$ ) then cooled, poured into saturated $\mathrm{NH}_{4} \mathrm{Cl}$ solution $(100 \mathrm{~mL})$ and extracted with $\mathrm{CHCl}_{3}(3 \times 25 \mathrm{ml})$. The combined organic extracts were washed with $\mathrm{H}_{2} \mathrm{O}(2 \times 50 \mathrm{~mL})$, then brine $(50 \mathrm{~mL})$, and dried $\left(\mathrm{Na}_{2} \mathrm{SO}_{4}\right)$. The solvent was evaporated (reduced pressure) to afford a crude residue, which was purified by filtration through a small silica gel column using EtOAc-hexane as eluent.

3-(3,4-Dimethoxyphenylamino)-3-methylthio-1-\{2-(4-methoxyphenyl)cyclopropyl $\}$ -

propenone (3a). Yield $2.80 \mathrm{~g}(70 \%)$; white solid, mp. $122-123{ }^{\circ} \mathrm{C} ; \mathrm{R}_{f} 0.50$ (1:4 EtOAc-hexane); IR (KBr): 2956, 2836, 1589, 1550, 1515, 1465, 1246, $1020 \mathrm{~cm}^{-1} ;{ }^{1} \mathrm{H}$ NMR $\left(\mathrm{CDCl}_{3}, 400 \mathrm{MHz}\right): \delta$ 12.73 (s, 1H, NH), 7.04 (d, $J=8.7 \mathrm{~Hz}, 2 \mathrm{H}, \operatorname{ArH}), 6.83-6.79(\mathrm{~m}, 4 \mathrm{H}, \mathrm{ArH}), 6.75$ (d, $J=1.7 \mathrm{~Hz}$, $1 \mathrm{H}, \mathrm{ArH}), 5.29(\mathrm{~s}, 1 \mathrm{H}, \mathrm{CH}), 3.86\left(\mathrm{~s}, 3 \mathrm{H}, \mathrm{OCH}_{3}\right), 3.83\left(\mathrm{~s}, 3 \mathrm{H}, \mathrm{OCH}_{3}\right), 3.76\left(\mathrm{~s}, 3 \mathrm{H}, \mathrm{OCH}_{3}\right), 2.47$ (ddd, $J=9.2 \mathrm{~Hz}, 5.9 \mathrm{~Hz}, 3.7 \mathrm{~Hz}, 1 \mathrm{H}, \mathrm{CH}), 2.30$ (s, 3H, $\left.\mathrm{SCH}_{3}\right), 1.91$ (ddd, J=7.9 Hz, $4.3 \mathrm{~Hz}, 4.3$ $\mathrm{Hz}, 1 \mathrm{H}, \mathrm{CH}$ ), 1.65 (ddd, $J=9.2 \mathrm{~Hz}, 4.6 \mathrm{~Hz}, 4.6 \mathrm{~Hz}, 1 \mathrm{H}, \mathrm{CH}$ ), 1.25 (ddd, J=8.1 Hz, $6.4 \mathrm{~Hz}, 4.2$ $\mathrm{Hz}, 1 \mathrm{H}, \mathrm{CH}) ;{ }^{13} \mathrm{C} \mathrm{NMR}\left(\mathrm{CDCl}_{3}, 100 \mathrm{MHz}\right): \delta 192.6,166.1,158.0,148.9,147.6,133.5,131.0$, 127.0, 117.8, 113.8, 110.7, 109.4, 91.4, 55.91, 55.90, 55.3, 32.7, 26.7, 17.3, 14.6. Anal. Calcd. for $\mathrm{C}_{22} \mathrm{H}_{25} \mathrm{NO}_{4} \mathrm{~S}$ (399.50): C, 66.14; H, 6.31; N, 3.51\%. Found: C, 66.12; H, 6.30; N, 3.48\%.

3-Methylthio-3-(m-tolylamino)-1-\{2-(4-methoxyphenyl)cyclopropyl\}propenone (3i). Yield 88\% (3.10g); white solid; mp. 96-97 ${ }^{\circ} \mathrm{C}$; R 0.50 (1:9 EtOAc-hexane); IR (KBr): 2995, 2926, 1584, 1541, 1466, 1243, $1148 \mathrm{~cm}^{-1} ;{ }^{1} \mathrm{H} \mathrm{NMR}\left(\mathrm{CDCl}_{3}, 400 \mathrm{MHz}\right): \delta 12.95$ (s, 1H, NH), 7.24 (t, $J$ $=7.6 \mathrm{~Hz}, 1 \mathrm{H}, \mathrm{ArH}), 7.12-7.07(\mathrm{~m}, 4 \mathrm{H}, \mathrm{ArH}), 7.04(\mathrm{~d}, J=7.6 \mathrm{~Hz}, 1 \mathrm{H}, \mathrm{ArH}), 6.85(\mathrm{~d}, J=8.8 \mathrm{~Hz}$, 2H, ArH), 5.37 (s, 1H, CH), $3.79\left(\mathrm{~s}, 3 \mathrm{H}, \mathrm{OCH}_{3}\right), 2.54$ (ddd, $\left.J=9.3 \mathrm{~Hz}, 6.0 \mathrm{~Hz}, 3.7 \mathrm{~Hz}, 1 \mathrm{H}, \mathrm{CH}\right)$, $2.35\left(\mathrm{~s}, 3 \mathrm{H}, \mathrm{CH}_{3}\right), 2.33\left(\mathrm{~s}, 3 \mathrm{H}, \mathrm{SCH}_{3}\right), 1.96(\mathrm{ddd}, J=8.1 \mathrm{~Hz}, 4.4 \mathrm{~Hz}, 4.4 \mathrm{~Hz}, 1 \mathrm{H}, \mathrm{CH}), 1.72$ (ddd, $J=9.0 \mathrm{~Hz}, 5.0 \mathrm{~Hz}, 4.2 \mathrm{~Hz}, 1 \mathrm{H}, \mathrm{CH}), 1.30(\mathrm{ddd}, J=9.3 \mathrm{~Hz}, 5.1 \mathrm{~Hz}, 2.5 \mathrm{~Hz}, 1 \mathrm{H}, \mathrm{CH}) ;{ }^{13} \mathrm{C} \mathrm{NMR}$ $\left(\mathrm{CDCl}_{3}, 100 \mathrm{MHz}\right): \delta 192.5,165.0,157.9,138.8,138.0,133.4,128.6,126.9,126.7,125.4,121.8$, 113.7, 91.6, 55.1, 32.6, 26.6, 21.2, 17.2, 14.4. Anal. Calcd. for $\mathrm{C}_{21} \mathrm{H}_{23} \mathrm{NO}_{2} \mathrm{~S}$ (353.48): C, 71.36; H, 6.56; N, 3.96\%. Found: C, 71.32; H, 6.54; N, 3.94\%.

General procedure for PPA- induced cyclization of $N, S$-arylaminoacetals (3a-k) to cyclopenta $[c]$ quinolines (6a-k)

A mixture of $N, S$-arylaminoacetals $3(1 \mathrm{mmol})$ and freshly prepared PPA $(10 \mathrm{~g})$ was heated at 90 ${ }^{\circ} \mathrm{C}$ for $6-10 \mathrm{~h}$. The reaction mixture was cooled, neutralized with sat. $\mathrm{NaHCO}_{3}$ solution and the aqueous layer was extracted with $\mathrm{CH}_{2} \mathrm{Cl}_{2}(3 \times 20 \mathrm{ml})$. The combined extracts were washed with $\mathrm{H}_{2} \mathrm{O}(3 \times 30 \mathrm{~mL})$, brine $(30 \mathrm{~mL})$, dried $\left(\mathrm{Na}_{2} \mathrm{SO}_{4}\right)$ and evaporated under reduced pressure to give crude products 6 which were purified on a small silica gel column using EtOAc/hexane as eluent.

\section{7,8-Dimethoxy-4-methylthio-3-(4-methoxyphenyl)-2,3-dihydro-1H-cyclopenta[c]quinoline}

(6a). Yield 0.20 g (54\%); colorless solid; mp. 192-193 ${ }^{\circ} \mathrm{C} ; \mathrm{R}_{f} 0.40$ (1:4 EtOAc-hexane); IR (KBr): 2929, 1617, 1582, 1507, $1241 \mathrm{~cm}^{-1} ;{ }^{1} \mathrm{H} \mathrm{NMR}\left(\mathrm{CDCl}_{3}, 400 \mathrm{MHz}\right): \delta 7.40$ (s, $\left.1 \mathrm{H}, \mathrm{ArH}\right)$, $6.98(\mathrm{~s}, 1 \mathrm{H}, \mathrm{ArH}), 6.96$ (d, $J=8.6 \mathrm{~Hz}, 2 \mathrm{H}, \mathrm{ArH}), 6.78(\mathrm{~d}, J=8.5 \mathrm{~Hz}, 2 \mathrm{H}, \mathrm{ArH}), 4.46(\mathrm{dd}, J=9.0$ $\mathrm{Hz}, 3.2 \mathrm{~Hz}, 1 \mathrm{H}, \mathrm{CH}), 4.02\left(\mathrm{~s}, 3 \mathrm{H}, \mathrm{OCH}_{3}\right), 4.0$ (s, 3H, $\left.\mathrm{OCH}_{3}\right), 3.75$ (s, 3H, $\left.\mathrm{OCH}_{3}\right), 3.29$ (ddd, $J=$ $\left.16.4 \mathrm{~Hz}, 8.4 \mathrm{~Hz}, 8.3 \mathrm{~Hz}, 1 \mathrm{H}, \mathrm{CH}_{2}\right), 3.14$ (ddd, $\left.J=16.6 \mathrm{~Hz}, 9.3 \mathrm{~Hz}, 3.6 \mathrm{~Hz}, 1 \mathrm{H}, \mathrm{CH}_{2}\right), 2.74-2.67$ $\left(\mathrm{m}, 1 \mathrm{H}, \mathrm{CH}_{2}\right), 2.56\left(\mathrm{~s}, 3 \mathrm{H}, \mathrm{SCH}_{3}\right), 2.17-2.10\left(\mathrm{~m}, 1 \mathrm{H}, \mathrm{CH}_{2}\right) ;{ }^{13} \mathrm{C} \mathrm{NMR}\left(\mathrm{CDCl}_{3}, 100 \mathrm{MHz}\right): \delta$ $158.0,154.6,151.6,148.4,148.1,144.5,136.3,135.0,128.4,118.6,113.7,107,5,102.5,56.04$, 
56.98, 55.0, 49.3, 35.4, 29.9, 12.7; $\mathrm{MS}(\mathrm{m} / \mathrm{z}, \%): 382\left(\mathrm{M}^{+}+1,77\right), 154$ (100); Anal. Calcd. for $\mathrm{C}_{22} \mathrm{H}_{23} \mathrm{NO}_{3} \mathrm{~S}$ (381.49): C, 69.26; H, 6.08; N, 3.67\%. Found: C, 69.24; H, 6.03; N, 3.69\%.

7-Methoxy-4-methylthio-3-(4-methoxyphenyl)-2,3-dihydro-1H-cyclopenta[c]quinoline (6b). Yield 56\% (0.20 g). White solid; mp. $162-163{ }^{\circ} \mathrm{C} ; \mathrm{R}_{f} 0.50$ (1:9 EtOAc-hexane); IR (KBr): 2927 , $1617,1586,1508,1413,1225 \mathrm{~cm}^{-1} ;{ }^{1} \mathrm{H} \mathrm{NMR}\left(\mathrm{CDCl}_{3}, 400 \mathrm{MHz}\right): \delta 7.68(\mathrm{~d}, J=8.8 \mathrm{~Hz}, 1 \mathrm{H}$, $\operatorname{ArH}), 7.36(\mathrm{~d}, J=2.4 \mathrm{~Hz}, 1 \mathrm{H}, \mathrm{ArH}), 7.11(\mathrm{dd}, J=8.7 \mathrm{~Hz}, 2.4 \mathrm{~Hz}, 1 \mathrm{H}, \mathrm{ArH}), 6.98$ (d, $J=8.8 \mathrm{~Hz}$, 2H, ArH), 6.80 (d, $J=8.5 \mathrm{~Hz}, 2 \mathrm{H}, \mathrm{ArH}), 4.48$ (dd, $J=8.9 \mathrm{~Hz}, 3.3 \mathrm{~Hz}, 1 \mathrm{H}, \mathrm{CH}), 3.96$ (s, $3 \mathrm{H}$, $\left.\mathrm{OCH}_{3}\right), 3.77\left(\mathrm{~s}, 3 \mathrm{H}, \mathrm{OCH}_{3}\right), 3.34\left(\mathrm{ddd}, J=16.9 \mathrm{~Hz}, 8.4 \mathrm{~Hz}, 8.4 \mathrm{~Hz}, 1 \mathrm{H}, \mathrm{CH}_{2}\right), 3.2$ (ddd, $J=16.8$ $\left.\mathrm{Hz}, 9.3 \mathrm{~Hz}, 3.9 \mathrm{~Hz}, 1 \mathrm{H}, \mathrm{CH}_{2}\right), 2.79-2.69\left(\mathrm{~m}, 1 \mathrm{H}, \mathrm{CH}_{2}\right), 2.58\left(\mathrm{~s}, 3 \mathrm{H}, \mathrm{SCH}_{3}\right), 2.18-2.11(\mathrm{~m}, 1 \mathrm{H}$, $\left.\mathrm{CH}_{2}\right) ;{ }^{13} \mathrm{C} \mathrm{NMR}\left(\mathrm{CDCl}_{3}, 100 \mathrm{MHz}\right): \delta 160.3,158.1,149.5,149.1,136.4,134.7,128.4,125.5$, 118.8, 117.4, 113.8, 107.0, 55.5, 55.1, 49.3, 35.5, 29.8, 12.6; $\mathrm{MS}(\mathrm{m} / \mathrm{z}, \%): 352\left(\mathrm{M}^{+}+1,100\right)$; Anal. Calcd. for $\mathrm{C}_{21} \mathrm{H}_{21} \mathrm{NO}_{2} \mathrm{~S}$ (351.46): C, 71.76; H, 6.02; N, 3.99\%. Found: C, 71.72; H, 6.04; N, $4.00 \%$.

7-Methoxy-4-methylthio-3-phenyl-2,3-dihydro-1H-cyclopenta[c]quinoline (6c). Yield 55\% (0.18 g); brown solid; mp. 182-183 ${ }^{\circ} \mathrm{C}$; $\mathrm{R}_{f} 0.6$ (1:9 EtOAc-hexane); IR (KBr): 2913, 1616, 1586, 1500, 1415, 1303, 1227, $1148 \mathrm{~cm}^{-1}$; ${ }^{1} \mathrm{H} \mathrm{NMR}\left(\mathrm{CDCl}_{3}, 400 \mathrm{MHz}\right): \delta 7.67(\mathrm{~d}, J=9.0 \mathrm{~Hz}, 1 \mathrm{H}$, ArH), 7.28-7.18 (m, 4H, ArH), 7.12 (dd, $J=9.0 \mathrm{~Hz}, 2.4 \mathrm{~Hz}, 1 \mathrm{H}, \mathrm{ArH}), 7.07$ (d, $J=1.4 \mathrm{~Hz}, 1 \mathrm{H}$, ArH), 7.05 (d, $J=8.7 \mathrm{~Hz}, 1 \mathrm{H}, \mathrm{ArH}), 4.5$ (dd, $J=9.3 \mathrm{~Hz}, 3.2 \mathrm{~Hz}, 1 \mathrm{H}, \mathrm{CH}), 3.95$ (s, 3H, OCH $)_{\text {, }}$ 3.35 (ddd, $\left.J=16.9 \mathrm{~Hz}, 8.4 \mathrm{~Hz}, 8.4 \mathrm{~Hz}, 1 \mathrm{H}, \mathrm{CH}_{2}\right), 3.20$ (ddd, $J=17.0 \mathrm{~Hz}, 9.3 \mathrm{~Hz}, 3.8 \mathrm{~Hz}, 1 \mathrm{H}$, $\left.\mathrm{CH}_{2}\right), 2.81-2.73\left(\mathrm{~m}, 1 \mathrm{H}, \mathrm{CH}_{2}\right), 2.61\left(\mathrm{~s}, 3 \mathrm{H}, \mathrm{SCH}_{3}\right), 2.21-2.15\left(\mathrm{~m}, 1 \mathrm{H}, \mathrm{CH}_{2}\right) ;{ }^{13} \mathrm{C} \mathrm{NMR}\left(\mathrm{CDCl}_{3}\right.$, $100 \mathrm{MHz}$ ): $\delta 160.8,157.7,157.6,144.0,134.4,128.5,127.4,126.5,125.5,118.7,117.8,117.7$, 106.4, 55.6, 50.1, 35.3, 29.9, 13.0; MS (m/z, \%): $322\left(\mathrm{M}^{+}+1,100\right)$; Anal. Calcd. for $\mathrm{C}_{20} \mathrm{H}_{19} \mathrm{NOS}$ (321.44): C, 74.73; H, 5.96; N, 4.36\%. Found: C, 74.70; H, 5.98; N, 4.33\%.

7-Methoxy-4-methylthio-3-(2,5-dimethoxyphenyl-2,3-dihydro-1H-cyclopenta[c]quinoline

(6d). Yield 54\% (0.21 g); colorless solid, mp. 132-133 ${ }^{\circ} \mathrm{C} ; \mathrm{R}_{f} 0.6$ (1:6 EtOAc-hexane); IR (KBr): 2930, 2830, 1618, 1586, 1499, 1485, 1418, 1280, $1219 \mathrm{~cm}^{-1} ;{ }^{1} \mathrm{H} \mathrm{NMR}\left(\mathrm{CDCl}_{3}, 400\right.$ $\mathrm{MHz}): \delta 7.65$ (d, $J=8.8 \mathrm{~Hz}, 1 \mathrm{H}, \operatorname{ArH}), 7.37$ (d, $J=2.4 \mathrm{~Hz}, 1 \mathrm{H}, \mathrm{ArH}), 7.10$ (dd, $J=8.9 \mathrm{~Hz}, 2.6$ $\mathrm{Hz}, 1 \mathrm{H}, \mathrm{ArH}), 6.85(\mathrm{~d}, J=8.8 \mathrm{~Hz}, 1 \mathrm{H}, \mathrm{ArH}), 6.70(\mathrm{dd}, J=8.8 \mathrm{~Hz}, 3.2 \mathrm{~Hz}, 1 \mathrm{H}, \mathrm{ArH}), 6.17(\mathrm{~d}, J=$ $3.2 \mathrm{~Hz}, 1 \mathrm{H}, \mathrm{ArH}), 4.85(\mathrm{dd}, J=9.3 \mathrm{~Hz}, 2.7 \mathrm{~Hz}, 1 \mathrm{H}, \mathrm{CH}), 3.96\left(\mathrm{~s}, 3 \mathrm{H}, \mathrm{OCH}_{3}\right), 3.85(\mathrm{~s}, 3 \mathrm{H}$, $\left.\mathrm{OCH}_{3}\right), 3.60\left(\mathrm{~s}, 3 \mathrm{H}, \mathrm{OCH}_{3}\right), 3.25-3.14\left(\mathrm{~m}, 2 \mathrm{H}, \mathrm{CH}_{2}\right), 2.73-2.68\left(\mathrm{~m}, 1 \mathrm{H}, \mathrm{CH}_{2}\right), 2.59(\mathrm{~s}, 3 \mathrm{H}$, $\left.\mathrm{SCH}_{3}\right), 2.14-2.07\left(\mathrm{~m}, 1 \mathrm{H}, \mathrm{CH}_{2}\right) ;{ }^{13} \mathrm{C} \mathrm{NMR}\left(\mathrm{CDCl}_{3}, 100 \mathrm{MHz}\right): \delta 160.3,157.6,153.5,151.4$, 150.0, 149.5, 133.9, 133.6, 125.4, 118.9, 117.3, 114.5, 111.6, 110.7, 106.9, 56.3, 55.5, 55.4, 43.2, 33.9, 29.7, 12.5; MS (m/z, \%): $382\left(\mathrm{M}^{+}+1,100\right)$; Anal. Calcd. for $\mathrm{C}_{22} \mathrm{H}_{23} \mathrm{NO}_{3} \mathrm{~S}$ (381.49): C, 69.26; H, 6.08; N, 3.67\%. Found: C, 69.21; H, 6.12; N, 3.69\%.

6,9-Dimethoxy-4-methylthio-3-phenyl-2,3-dihydro-1H-cyclopenta[c]quinoline (6e). Yield 51\% (0.18 g); brown solid; mp. 120-121 ${ }^{\circ} \mathrm{C}$; $\mathrm{R}_{f} 0.4$ (1:9 EtOAc-hexane); IR (KBr): 2928, 1607 , 1584, 1560, 1465, 1258, $1107 \mathrm{~cm}^{-1} ;{ }^{1} \mathrm{H} \mathrm{NMR}\left(\mathrm{CDCl}_{3}, 400 \mathrm{MHz}\right): \delta 7.23(\mathrm{t}, J=6.8 \mathrm{~Hz}, 2 \mathrm{H}$, $\operatorname{ArH}), 7.18(\mathrm{t}, J=7.2 \mathrm{~Hz}, 1 \mathrm{H}, \mathrm{ArH}), 7.03$ (d, $J=7.0 \mathrm{~Hz}, 2 \mathrm{H}, \mathrm{ArH}), 6.94(\mathrm{~d}, J=8.6 \mathrm{~Hz}, 1 \mathrm{H}$, $\operatorname{ArH}), 6.67$ (d, $J=8.6 \mathrm{~Hz}, 1 \mathrm{H}, \mathrm{ArH}), 4.46(\mathrm{dd}, J=9.5 \mathrm{~Hz}, 2.7 \mathrm{~Hz}, 1 \mathrm{H}, \mathrm{CH}), 4.02\left(\mathrm{~s}, 3 \mathrm{H}, \mathrm{OCH}_{3}\right)$, $3.89\left(\mathrm{~s}, 3 \mathrm{H}, \mathrm{OCH}_{3}\right), 3.62-3.58\left(\mathrm{~m}, 2 \mathrm{H}, \mathrm{CH}_{2}\right), 2.70-2.62\left(\mathrm{~m}, 4 \mathrm{H}, \mathrm{SCH}_{3}+1 \mathrm{H}, \mathrm{CH}_{2}\right), 2.14-2.07$ 
$\left(\mathrm{m}, 1 \mathrm{H}, \mathrm{CH}_{2}\right) ;{ }^{13} \mathrm{C} \mathrm{NMR}\left(\mathrm{CDCl}_{3}, 100 \mathrm{MHz}\right): \delta 157.0,150.8,149.1,148.9,144.2,140.8,136.8$, $128.4,127.4,126.3,117.6,108.6,103.1,56.9,55.5,48.9,35.3,34.1,12.4 ; \mathrm{MS}(\mathrm{m} / \mathrm{z}, \%): 352$ $\left(\mathrm{M}^{+}+1,100\right)$; Anal. Calcd. for $\mathrm{C}_{21} \mathrm{H}_{21} \mathrm{NO}_{2} \mathrm{~S}$ (351.46): C, 71.76; H, 6.02; N, 3.99\%. Found: C, $71.71 ; \mathrm{H}, 6.07 ; \mathrm{N}, 4.00 \%$.

\section{6,9-Dimethoxy-4-methylthio-3-(3,4-dimethoxyphenyl)-2,3-dihydro-1H-cyclopenta[c]-}

quinoline (6f). Yield 54\% (0.22 g); white solid; mp. 144-145 ${ }^{\circ} \mathrm{C}$; $\mathrm{R}_{f} 0.4$ (1:3 EtOAc-hexane); IR (KBr): 2945, 2932, 1585, 1563, 1498, $1218 \mathrm{~cm}^{-1} ;{ }^{1} \mathrm{H}$ NMR $\left(\mathrm{CDCl}_{3}, 400 \mathrm{MHz}\right): \delta 6.94(\mathrm{~d}, J=8.6$ $\mathrm{Hz}, 1 \mathrm{H}, \mathrm{ArH}), 6.72(\mathrm{~d}, J=8.3 \mathrm{~Hz}, 1 \mathrm{H}, \mathrm{ArH}), 6.68(\mathrm{~d}, J=8.5 \mathrm{~Hz}, 1 \mathrm{H}, \operatorname{ArH}), 6.60(\mathrm{~d}, J=1.9 \mathrm{~Hz}$, 1H, ArH), 6.54 (dd, $J=8.3 \mathrm{~Hz}, 2.0 \mathrm{~Hz}, 1 \mathrm{H}, \mathrm{ArH}), 4.42$ (dd, $J=9.3 \mathrm{~Hz}, 3.2 \mathrm{~Hz}, 1 \mathrm{H}, \mathrm{CH}), 4.02$ (s, $\left.3 \mathrm{H}, \mathrm{OCH}_{3}\right), 3.90\left(\mathrm{~s}, 3 \mathrm{H}, \mathrm{OCH}_{3}\right), 3.83\left(\mathrm{~s}, 3 \mathrm{H}, \mathrm{OCH}_{3}\right), 3.78\left(\mathrm{~s}, 3 \mathrm{H}, \mathrm{OCH}_{3}\right), 3.60-3.57(\mathrm{~m}, 2 \mathrm{H}$, $\left.\mathrm{CH}_{2}\right), 2.71-2.61\left(\mathrm{~m}, 4 \mathrm{H}, \mathrm{SCH}_{3}+1 \mathrm{H}, \mathrm{CH}_{2}\right), 2.12-2.05\left(\mathrm{~m}, 1 \mathrm{H}, \mathrm{CH}_{2}\right) ;{ }^{13} \mathrm{C} \mathrm{NMR}\left(\mathrm{CDCl}_{3}, 100\right.$ $\mathrm{MHz}): \delta 157.1,150.8,149.1,149.0,147.5,140.7,136.9,136.8,119.3,117.6,110.84,110.8$, 108.5, 103.1, 56.8, 55.8, 55.7, 55.5, 48.7, 35.5, 34.0, 12.5; MS (m/z, \%): $412\left(\mathrm{M}^{+}+1,100\right)$; Anal. Calcd. for $\mathrm{C}_{23} \mathrm{H}_{25} \mathrm{NO}_{4} \mathrm{~S}$ (411.51): C, 67.13; H, 6.12; N, 3.40\%. Found: C, 67.10; H, 6.16; N, $3.44 \%$.

8-Methoxy-4-methylthio-3-(4-methoxyphenyl)-2,3-dihydro- $1 H$-cyclopenta[c]quinoline (6g). Yield 50\% (0.18 g); white solid; mp. $152-153{ }^{\circ} \mathrm{C}$; $\mathrm{R}_{f} 0.60$ (1:9 EtOAc-hexane); IR (KBr): 2921, $1617,1576,1550,1508,1458,1242,1031 \mathrm{~cm}^{-1} ;{ }^{1} \mathrm{H}$ NMR $\left(\mathrm{CDCl}_{3}, 400 \mathrm{MHz}\right): \delta 7.93(\mathrm{~d}, J=7.3$ $\mathrm{Hz}, 1 \mathrm{H}, \mathrm{ArH}), 7.29$ (dd, $J=9.0 \mathrm{~Hz}, 2.7 \mathrm{~Hz}, 1 \mathrm{H}, \mathrm{ArH}), 7.03$ (d, $J=2.7 \mathrm{~Hz}, 1 \mathrm{H}, \mathrm{ArH}), 6.98$ (dd, $J$ $=8.5 \mathrm{~Hz}, 1.7 \mathrm{~Hz}, 2 \mathrm{H}, \mathrm{ArH}), 6.80(\mathrm{dd}, J=8.5 \mathrm{~Hz}, 1.7 \mathrm{~Hz}, 2 \mathrm{H}, \mathrm{ArH}), 4.49$ (dd, $J=9.0,3.2 \mathrm{~Hz}$, $1 \mathrm{H}, \mathrm{CH}), 3.93\left(\mathrm{~s}, 3 \mathrm{H}, \mathrm{OCH}_{3}\right), 3.76\left(\mathrm{~s}, 3 \mathrm{H}, \mathrm{OCH}_{3}\right), 3.60-3.30\left(\mathrm{~m}, 1 \mathrm{H}, \mathrm{CH}_{2}\right), 3.18(\mathrm{ddd}, J=16.7$ $\left.\mathrm{Hz}, 9.4 \mathrm{~Hz}, 3.7 \mathrm{~Hz}, 1 \mathrm{H}, \mathrm{CH}_{2}\right), 2.78-2.72\left(\mathrm{~m}, 1 \mathrm{H}, \mathrm{CH}_{2}\right), 2.57\left(\mathrm{~s}, 3 \mathrm{H}, \mathrm{SCH}_{3}\right), 2.19-2.14(\mathrm{~m}, 1 \mathrm{H}$, $\left.\mathrm{CH}_{2}\right) ;{ }^{13} \mathrm{C} \mathrm{NMR}\left(\mathrm{CDCl}_{3}, 100 \mathrm{MHz}\right): \delta 158.2,156.8,154.5,148.2,143.7,137.0,136.2,129.6$, $128.4,124.7,120.5,113.8,102.9,55.5,55.1,49.5,35.4,29.9,12.6$; $\mathrm{MS}(m / z, \%): 352\left(\mathrm{M}^{+}+1\right.$, 100), 154 (97); Anal. Calcd. for $\mathrm{C}_{21} \mathrm{H}_{21} \mathrm{NO}_{2} \mathrm{~S}$ (351.46): C, 71.76; H, 6.02; N, 3.99\%. Found: C, $71.78 ; \mathrm{H}, 6.00 ; \mathrm{N}, 3.96 \%$.

4-Methylthio-3-(4-methoxyphenyl)-2,3-dihydro-1H-cyclopenta[c]quinoline (6h). Yield 55\% (0.17 g); viscous liquid; $\mathrm{R}_{f} 0.70$ (1:9 EtOAc-hexane); IR (KBr): 2922, 1588, 1473, 1559, 1473, 1214, $1147 \mathrm{~cm}^{-1} ;{ }^{1} \mathrm{H}$ NMR $\left(\mathrm{CDCl}_{3}, 400 \mathrm{MHz}\right): \delta 7.92(\mathrm{~d}, J=8.3 \mathrm{~Hz}, 1 \mathrm{H}, \mathrm{ArH}), 7.71(\mathrm{~d}, J=8.0$ $\mathrm{Hz}, 1 \mathrm{H}, \mathrm{ArH}), 7.55$ (td, $J=7.6 \mathrm{~Hz}, 1.4 \mathrm{~Hz}, 1 \mathrm{H}, \mathrm{ArH}), 7.38$ (td, $J=6.95 \mathrm{~Hz}, 1.2 \mathrm{~Hz}, 1 \mathrm{H}, \mathrm{ArH})$, $6.91(\mathrm{dd}, J=7.8 \mathrm{~Hz}, 2.0 \mathrm{~Hz}, 2 \mathrm{H}, \mathrm{ArH}), 6.72(\mathrm{dd}, J=7.8 \mathrm{~Hz}, 2.2 \mathrm{~Hz}, 2 \mathrm{H}, \mathrm{ArH}), 4.43$ (d, $J=9.0$ $\mathrm{Hz}, 1 \mathrm{H}, \mathrm{CH}), 3.69$ (s, 3H, $\left.\mathrm{OCH}_{3}\right), 3.33-3.27\left(\mathrm{~m}, 1 \mathrm{H}, \mathrm{CH}_{2}\right), 3.18-3.16\left(\mathrm{~m}, 1 \mathrm{H}, \mathrm{CH}_{2}\right), 2.71-2.65$ $\left(\mathrm{m}, 1 \mathrm{H}, \mathrm{CH}_{2}\right), 2.51\left(\mathrm{~s}, 3 \mathrm{H}, \mathrm{SCH}_{3}\right), 2.09-206\left(\mathrm{~m}, 1 \mathrm{H}, \mathrm{CH}_{2}\right) ;{ }^{13} \mathrm{C} \mathrm{NMR}\left(\mathrm{CDCl}_{3}, 100 \mathrm{MHz}\right): \delta$ $158.2,157.5,149.0,147.8,136.7,136.2,128.7,128.5,128.2,124.9,124.4,124.0,113.8,55.1$, 49.5, 35.4, 29.8, 12.6; MS (m/z, \%): $322\left(\mathrm{M}^{+}+1,100\right), 154$ (80). Anal. Calcd. for $\mathrm{C}_{20} \mathrm{H}_{19} \mathrm{NOS}$ (321.44): C, 74.73; H, 5.96; N, 4.36\%. Found: C, 74.74; H, 5.92; N, 4.33\%.

7-Methyl-4-methylthio-3-(4-methoxyphenyl)-2,3-dihydro-1H-cyclopenta[c]quinoline (6i). Yield 46\% (0.15 g); colorless solid; mp. 126-127 ${ }^{\circ} \mathrm{C}$; $\mathrm{R}_{f} 0.60$ (1:9 EtOAc-hexane); IR (KBr): 2920, 1584, 1509, 1297, $1246 \mathrm{~cm}^{-1} ;{ }^{1} \mathrm{H} \mathrm{NMR}\left(\mathrm{CDCl}_{3}, 400 \mathrm{MHz}\right): \delta 7.78(\mathrm{~s}, 1 \mathrm{H}, \mathrm{ArH}), 7.60(\mathrm{~d}, J$ $=8.3 \mathrm{~Hz}, 1 \mathrm{H}, \mathrm{ArH}), 7.23(\mathrm{dd}, J=8.3 \mathrm{~Hz}, 1.2 \mathrm{~Hz}, 1 \mathrm{H}, \mathrm{ArH}), 6.91(\mathrm{~d}, J=8.5 \mathrm{~Hz}, 2 \mathrm{H}, \mathrm{ArH}), 6.73$ 
(d, $J=8.8 \mathrm{~Hz}, 2 \mathrm{H}, \mathrm{ArH}), 4.42(\mathrm{dd}, J=9.0 \mathrm{~Hz}, 2.9 \mathrm{~Hz}, 1 \mathrm{H}, \mathrm{CH}), 3.70$ (s, 3H, OCH ), 3.29 (ddd, $J$ $\left.=16.8 \mathrm{~Hz}, 8.5 \mathrm{~Hz}, 8.4 \mathrm{~Hz}, 1 \mathrm{H}, \mathrm{CH}_{2}\right), 3.13\left(\mathrm{ddd}, J=16.9 \mathrm{~Hz}, 9.3 \mathrm{~Hz}, 3.8 \mathrm{~Hz}, 1 \mathrm{H}, \mathrm{CH}_{2}\right), 2.72-$ $2.62\left(\mathrm{~m}, 1 \mathrm{H}, \mathrm{CH}_{2}\right), 2.53\left(\mathrm{~s}, 3 \mathrm{H}, \mathrm{SCH}_{3}\right), 2.48\left(\mathrm{~s}, 3 \mathrm{H}, \mathrm{CH}_{3}\right), 2.12-2.05\left(\mathrm{~m}, 1 \mathrm{H}, \mathrm{CH}_{2}\right) ;{ }^{13} \mathrm{C} \mathrm{NMR}$ $\left(\mathrm{CDCl}_{3}, 100 \mathrm{MHz}\right): \delta 158.0,157.3,149.0,147.8,139.0,136.2,135.9,128.4,127.2,127.0,124.0$, 121.8, 113.8, 55.0, 49.4, 35.4, 29.8, 21.8, 12.6; MS (m/z, \%): $336\left(\mathrm{M}^{+}+1,100\right)$. Anal. Calcd. for $\mathrm{C}_{21} \mathrm{H}_{21} \mathrm{NOS}$ (335.46): C, 75.19; H, 6.31; N, 4.18\%. Found: C, 75.15; H, 6.35; N, 4.21\%.

7-Chloro-4-methylthio-3-phenyl-2,3-dihydro-1H-cyclopenta[c]quinoline (6j). Yield 52\% (0.17 g); light yellow solid; mp. $132-133{ }^{\circ} \mathrm{C}$; $\mathrm{R}_{f} 0.60$ (1:8 EtOAc-hexane); IR (KBr): 2924, 1605, 1582, 1508, 1488, 1294, $1029 \mathrm{~cm}^{-1} ;{ }^{1} \mathrm{H}$ NMR $\left(\mathrm{CDCl}_{3}, 400 \mathrm{MHz}\right): \delta 8.01(\mathrm{~s}, 1 \mathrm{H}, \mathrm{ArH})$, $7.65(\mathrm{~d}, J=8.8 \mathrm{~Hz}, 1 \mathrm{H}, \mathrm{ArH}), 7.36$ (dd, $J=8.7 \mathrm{~Hz}, 1.6 \mathrm{~Hz}, 1 \mathrm{H}, \mathrm{ArH}), 7.24$ (t, $J=7.0 \mathrm{~Hz}, 2 \mathrm{H})$, $7.18(\mathrm{t}, J=6.6 \mathrm{~Hz}, 1 \mathrm{H}, \mathrm{ArH}), 7.03$ (d, $J=7.8 \mathrm{~Hz}, 2 \mathrm{H}, \mathrm{ArH}), 4.48$ (dd, $J=9.0 \mathrm{~Hz}, 2.9 \mathrm{~Hz}, 1 \mathrm{H}$, $\mathrm{CH}$ ), 3.34 (ddd, $J=16.8 \mathrm{~Hz}, 8.4 \mathrm{~Hz}, 8.3 \mathrm{~Hz}, 1 \mathrm{H}, \mathrm{CH}_{2}$ ), 3.17 (ddd, $J=17.0 \mathrm{~Hz}, 9.4 \mathrm{~Hz}, 3.8 \mathrm{~Hz}$, $\left.1 \mathrm{H}, \mathrm{CH}_{2}\right), 2.80-2.70\left(\mathrm{~m}, 1 \mathrm{H}, \mathrm{CH}_{2}\right), 2.54\left(\mathrm{~s}, 3 \mathrm{H}, \mathrm{SCH}_{3}\right), 2.21-2.13\left(\mathrm{~m}, 1 \mathrm{H}, \mathrm{CH}_{2}\right)$; ${ }^{13} \mathrm{C}$ NMR $\left(\mathrm{CDCl}_{3}, 100 \mathrm{MHz}\right): \delta 159.0,149.5,147.6,143.6,136.7,134.7,128.5,127.4,126.8,126.6,125.8$, 125.6, 122.2, 50.2, 35.1, 29.9, 12.9; $\mathrm{MS}(\mathrm{m} / \mathrm{z}, \%): 327\left(\mathrm{M}^{+}+1,64\right), 326\left(\mathrm{M}^{+}, 100\right)$; Anal. Calcd. for $\mathrm{C}_{19} \mathrm{H}_{16} \mathrm{ClNS}$ (325.86): C, 70.03; H, 4.95; N, 4.30\%. Found: C, 70.06; H, 4.93; N, 4.27\%.

\section{6-Methylthio-7-(4-methoxyphenyl)-8,9-dihydro-7H-benzo $[\boldsymbol{h}]$ cyclopenta[c]quinoline (6k).}

Yield 48\% (0.18 g); colorless solid; mp. 102-103 ${ }^{\circ} \mathrm{C}$; $\mathrm{R}_{f} 0.70$ (1:7 EtOAc-hexane); IR (KBr): 2924, 1582, 1508, 1431, 1245, $1029 \mathrm{~cm}^{-1} ;{ }^{1} \mathrm{H} \mathrm{NMR}\left(\mathrm{CDCl}_{3}, 400 \mathrm{MHz}\right): \delta 9.23(\mathrm{~d}, J=8.1 \mathrm{~Hz}$, 1H, ArH), 7.82 (dd, $J=8.0 \mathrm{~Hz}, 1.4 \mathrm{~Hz}, 1 \mathrm{H}, \mathrm{ArH}), 7.69$ (d, $J=8.8 \mathrm{~Hz}, 1 \mathrm{H}, \mathrm{ArH}), 7.64(\mathrm{~d}, J=8.8$ $\mathrm{Hz}, 1 \mathrm{H}, \mathrm{ArH}), 7.58$ (t, $J=7.9 \mathrm{~Hz}, 2 \mathrm{H}, \mathrm{ArH}), 6.94(\mathrm{~d}, J=8.8 \mathrm{~Hz}, 2 \mathrm{H}, \mathrm{ArH}), 6.74(\mathrm{~d}, J=8.8 \mathrm{~Hz}$, 2H, ArH), $4.47(\mathrm{dd}, J=9.0 \mathrm{~Hz}, 3.4 \mathrm{~Hz}, 1 \mathrm{H}, \mathrm{CH}), 3.69$ (s, 3H, $\left.\mathrm{OCH}_{3}\right), 3.36$ (ddd, $J=18.7 \mathrm{~Hz}$, $10.3 \mathrm{~Hz}, 8.3 \mathrm{~Hz}, 1 \mathrm{H}, \mathrm{CH}_{2}$ ), 3.21 (ddd, $\left.J=16.8 \mathrm{~Hz}, 9.2 \mathrm{~Hz}, 4.0 \mathrm{~Hz}, 1 \mathrm{H}, \mathrm{CH}_{2}\right), 2.80-2.67$ (m, 4H, $\left.\mathrm{SCH}_{3}+1 \mathrm{H} \quad \mathrm{CH}_{2}\right), \quad 2.17-2.09\left(\mathrm{~m}, \quad 1 \mathrm{H}, \quad \mathrm{CH}_{2}\right) ;{ }^{13} \mathrm{C} \quad \mathrm{NMR} \quad\left(\mathrm{CDCl}_{3}, \quad 100 \mathrm{MHz}\right)$ : $\delta 158.2,155.6,149.9,144.9,137.4,136.0,133.5,131.1,128.5,127.8,127.6,126.7,126.0,124.7$, 122.2, 120.9, 113.8, 55.1, 49.4, 35.6, 30.0, 13.1; MS (m/z, \%): $372\left(\mathrm{M}^{+}+1,86\right), 154$ (100); Anal. Calcd. for $\mathrm{C}_{24} \mathrm{H}_{21} \mathrm{NOS}$ (371.49): C, 77.59; H, 5.70; N, 3.77\%. Found: C, 77.57; H, 5.72; N, $3.74 \%$.

\section{General procedure for Raney Ni de-thiomethylation of quinolines 6 a and $6 \mathbf{j}$}

A suspension of quinoline (6a or $\mathbf{6 j})(1 \mathrm{mmol})$ and Raney Ni (W2) (ca. $1 \mathrm{~g})$ in ethanol (20 ml) was heated at reflux with stirring for $3 \mathrm{~h}$ (monitored by TLC). It was then filtered through a sintered glass funnel and washed with hot ethanol. The filtrate was concentrated to afford a viscous residue, which was purified by column chromatography over silica gel using EtOAchexane $(1: 3)$ as eluent to give the pure products $(\mathbf{7} \mathbf{a}, 7 \mathbf{j})$.

7,8-Dimethoxy-3-(4-methoxyphenyl)-2,3-dihydro-1H-cyclopenta[c]quinoline (7a). Yield 88\% (0.29 g); colorless solid; mp. $116-117{ }^{\circ} \mathrm{C}$; $\mathrm{R}_{f} 0.5$ (1:3, EtOAc:hexane); ${ }^{1} \mathrm{H}$ NMR $\left(\mathrm{CDCl}_{3}\right.$, $300 \mathrm{MHz}): \delta 8.37$ (s, 1H, ArH), $7.47(\mathrm{~s}, 1 \mathrm{H}, \mathrm{ArH}), 7.10$ (d, J=8.4 Hz, 2H, ArH), 7.03 (s, 1H, $\operatorname{ArH}), 6.86(\mathrm{~d}, J=8.4 \mathrm{~Hz}, 2 \mathrm{H}, \mathrm{ArH}), 4.55(\mathrm{t}, J=8.0 \mathrm{~Hz}, 1 \mathrm{H}, \mathrm{CH}), 4.04\left(\mathrm{~s}, 3 \mathrm{H}, \mathrm{OCH}_{3}\right), 4.03(\mathrm{~s}$, $\left.3 \mathrm{H}, \mathrm{OCH}_{3}\right), 3.80\left(\mathrm{~s}, 3 \mathrm{H}, \mathrm{OCH}_{3}\right), 3.37$ (ddd, $\left.J=16.5 \mathrm{~Hz}, 9.0 \mathrm{~Hz}, 4.1 \mathrm{~Hz}, 1 \mathrm{H}, \mathrm{CH}_{2}\right), 3.16$ (ddd, $J=$ 16.5 Hz, 8.1 Hz, 8.1 Hz, 1H, $\left.\mathrm{CH}_{2}\right), 2.84-2.72\left(\mathrm{~m}, 1 \mathrm{H}, \mathrm{CH}_{2}\right), 2.22-2.09\left(\mathrm{~m}, 1 \mathrm{H}, \mathrm{CH}_{2}\right) ;{ }^{13} \mathrm{C} \mathrm{NMR}$ 
$\left(\mathrm{CDCl}_{3}, 75 \mathrm{MHz}\right): \delta 158.3,151.7,149.8,148.5,145.9,144.0,137.8,137.0,128.7,121.1,114.0$, 108.5, 101.9, 56.03, 56.02, 55.3, 49.6, 36.2, 29.9; MS (ES, m/z, \%): $336\left(\mathrm{M}^{+}+1,100\right)$; Anal. Calcd. for $\mathrm{C}_{21} \mathrm{H}_{21} \mathrm{NO}_{3}$ (335.40): C, 75.20; H, 6.31; N, 4.18\%. Found: C, 75.26; H, 6.29; N, $4.13 \%$.

Procedure for ruthenium tetroxide oxidation of 7-chloro-4-methylthio-3-phenyl-2,3dihydro- $1 H$-cyclopenta[c]quinoline (6j)

To a solution of quinoline $\mathbf{6 j}(1.0 \mathrm{mmol})$ in $\mathrm{CCl}_{4}-\mathrm{CH}_{3} \mathrm{CN}(8 \mathrm{~mL}, 1: 1)$, sodium periodate (10 mmol) was added with vigorous stirring followed by addition of a solution of $\mathrm{RuCl}_{3} \cdot 3 \mathrm{H}_{2} \mathrm{O}(8$ mol. \%) in water $(4 \mathrm{~mL})$. Reaction mixture was stirred for $20 \mathrm{~min}$. (monitored by TLC) and worked-up by addition of water $(10 \mathrm{~mL})$ to dissolve the separated sodium iodate. The aqueous layer was extracted with $\mathrm{CH}_{2} \mathrm{Cl}_{2}(3 \times 20 \mathrm{~mL})$ and the combined extracts were washed with $\mathrm{H}_{2} \mathrm{O}$ $(3 \times 30 \mathrm{~mL})$, brine $(30 \mathrm{~mL})$, dried $\left(\mathrm{Na}_{2} \mathrm{SO}_{4}\right)$ and filtered through Celite. The solvent was evaporated under reduced pressure to give crude product $\mathbf{9 j}$, which was purified using a small silica gel column using EtOAc/hexane (1:3) as eluent.

7-Chloro-4-methanesulfonyl-3-phenyl-2,3-dihydro-1H-cyclopenta[c]quinoline, $\mathbf{9 j}$. Yield 68\% (0.24 g); colorless solid, mp. 174-175 ${ }^{\circ} \mathrm{C}$; $\mathrm{R}_{f} 0.6$ (1:3 EtOAc:hexane); IR (KBr): 2999 , 2919, 1606, 1314, 1337, 1073 $\mathrm{cm}^{-1}$; ${ }^{1} \mathrm{H}$ NMR $\left(\mathrm{CDCl}_{3}, 300 \mathrm{MHz}\right): \delta 8.25$ (s, 1H, ArH), 7.91 (d, $J$ $=8.7 \mathrm{~Hz}, 1 \mathrm{H}, \mathrm{ArH}), 7.69(\mathrm{~d}, J=8.7 \mathrm{~Hz}, 1 \mathrm{H}, \mathrm{ArH}), 7.26-7.16(\mathrm{~m}, 3 \mathrm{H}, \mathrm{ArH}), 6.97(\mathrm{~d}, J=6.9 \mathrm{~Hz}$, 2H, ArH), $5.32(\mathrm{~d}, J=9.0 \mathrm{~Hz}, 1 \mathrm{H}, \mathrm{CH}), 3.54-3.33\left(\mathrm{~m}, 2 \mathrm{H}, \mathrm{CH}_{2}\right), 2.94\left(\mathrm{~s}, 3 \mathrm{H}, \mathrm{CH}_{3}\right), 2.90-2.77$ $\left(\mathrm{m}, 1 \mathrm{H}, \mathrm{CH}_{2}\right), 2.37-2.31\left(\mathrm{~m}, 1 \mathrm{H}, \mathrm{CH}_{2}\right) ;{ }^{13} \mathrm{C} \mathrm{NMR}\left(\mathrm{CDCl}_{3}, 75 \mathrm{MHz}\right): \delta 156.2,155.2,146.5$, $144.4,136.4,136.0,130.2,129.4,128.5,127.3,126.7,125.9,125.4,49.6,40.2,35.2$, 29.6; MS (ES, $m / z, \%): 358\left(\mathrm{M}^{+}+1,100\right)$; Anal. Calcd. for $\mathrm{C}_{19} \mathrm{H}_{16} \mathrm{ClNO}_{2} \mathrm{~S}(357.85): \mathrm{C}, 63.77 ; \mathrm{H}, 4.51 ; \mathrm{N}$, 3.91\%. Found: C, 63.74; H, 4.53; N, 3.94\%.

\section{Acknowledgements}

SKSY thanks ICAR for financial assistance. AKY thanks CSIR for a Junior Research Fellowship. GSMS thanks IITK for a Research Fellowship. Financial assistance under DST project is also acknowledged.

\section{References}

1. (a) Michael, J. P. Nat. Prod. Rep. 1997, 14, 605. (b) Balasubramanian, M.; Keay, J. G. In Comprehensive Heterocyclic Chemistry II, Vol. 5, Katritzky, A. R.; Rees, C. W.; Scriven, E. F. V., Eds.; Pergamon Press: Oxford, 1996, Chap. 5.06, p 245.

2. (a) Spicer, J. A.; Gamage, S. A.; Atwell, G. J.; Finlay, G. J.; Baguley, B. C.; Denny, W. A. J. Med. Chem. 1997, 40, 1919. (b) Lyou, M. A.; Lawrence, S.; Williams, D. J.; Jackson, Y. A. J. Chem. Soc., Perkin Trans. 1, 1999, 437. 
3. (a) Mahata, P. K.; Venkatesh, C.; Syam Kumar, U. K.; Ila, H.; Junjappa, H. J. Org. Chem. 2003, 68, 3966 and references therein. (b) Panda, K.; Siddiqui, I.; Mahata, P. K.; Ila, H.; Junjappa, H. Synlett 2004, 449. (c) Katritzky, A. R.; Arend, M. J. Org. Chem. 1998, 63, 9989. (d) Banwell, M. G.; Lupton, D. W.; Ma, X.; Renner, J.; Sydnes, M. O. Org. Lett. 2004, 6, 2741. (e) Heesoon, L.; Jeeman, L.; Sung-II, Y. Arch. Pharm. Res. 2001, 24, 385. (f) Fuchs, J. R.; Funk, R. L. Org. Lett. 2005, 7, 677. (g) Mehta, B. K.; Yanagisawa, K.; Shiro, M.; Kotsuki, H. Org. Lett. 2003, 5, 1605. (h) Denton, D. A.; Smally, R. K.; Suschitzky, H.; J. Chem. Soc. 1964, 2421. (i) Jaroch, S.; Holscher, P.; Rehwinket, H.; Sulzle, D.; Burton, G.; Hillmann, M.; McDonald, F. M. Bioorg. Med. Chem. Lett. 2003, 13, 1981. (j) Eisch, J. J.; Gopal, H.; Kuo, C.T. J. Org. Chem.1978, 43, 2190. (k) Brown, R. J.; Carver, F. W. S.; Hollingsworth, B. L. J. Chem. Soc.1961, 4295.

4. (a) Tietze, L. F. Chem. Rev. 1996, 96, 115. (b) Tietze, L. F.; Beifuss, U. Angew. Chem. Int. Ed. 1993, 32, 131. (c) Thematic Issue, Frontiers in Organic Synthesis; Wender, P.A., guest editor, Chem. Rev. 1996, 96, 31.

5. (a) Patro, B.; Deb, B.; Ila, H.; Junjappa, H. J. Org. Chem. 1992, 57, 2257. (b) Patro, B.; Deb, B.; Ila, H.; Junjappa, H. Tetrahedron 1994, 50, 255. (c) Patra, P. K.; Patro, B.; Ila, H.; Junjappa, H. Tetrahedron Lett. 1993, 34, 3951. (d) Patra, P. K.; Sriram, V.; Ila, H.; Junjappa, H. Tetrahedron 1998, 54, 531. (e) Mohanta, P. K.; Peruncheralathan, S.; Ila, H.; Junjappa, H. J. Org. Chem. 2001, 66, 1503. (f) Syam Kumar, U. K.; Patra, P. K.; Ila, H.; Junjappa, H. J. Chem. Soc., Perkin Trans. 1 2000, 1547. (g) Nandi, S.; Syam Kumar, U. K.; Ila, H.; Junjappa, H. J. Org. Chem. 2002, 67, 4916. (h) Venkatesh, C.; Ila, H.; Junjappa, H. J. Org. Chem. 2002, 67, 9477. (i) Peruncheralathan, S.; Sriram, V. ; Ila, H.; Junjappa, H. Tetrahedron 2004, 60, 5603.

6. Deb, B.; Asokan, C. V.; Ila, H.; Junjappa, H. Tetrahedron Lett. 1988, 29, 2111.

7. Singh, O. M.; Junjappa, H.; Ila, H. J. Chem. Soc. Perkin Trans. 1 1997, 3561. 